\title{
Intraocular infestation by the reindeer warble fly larva: an unusual indication for acute vitrectomy
}

\author{
M GJÖTTERBERG AND S O INGEMANSSON \\ From the Department of Ophthalmology, Karolinska Institute, Huddinge University Hospital, \\ S-141 86 Huddinge, Sweden
}

SUMMARY A case of ophthalmomyiasis interna posterior (OIP) caused by the larva of the Reindeer warble fly is reported. The larva was initially found intraretinally in the inferior parts of the fundus. From this position it moved into the macula lutea within two days, causing decreased visual acuity. Acute vitrectomy was performed and the larva was removed through a retinotomy. About two months afterwards a small retinal detachment appeared near the ora serrata, where the larva had entered the eye. The retina was reattached with a scleral buckling procedure. The patient regained most of his vision postoperatively. Pars plana vitrectomy is a safe procedure and we consider that in any case of OIP, with a living larva in the eye, acute vitrectomy should be considered, since the larva may damage vital parts of the eye. Furthermore, the site of entrance should be treated with photocoagulation to prevent retinal detachment.

The reindeer is practically the only animal host for the reindeer warble fly (Oedemagena tarandi). However, it has been observed that the fly often deposits eggs in the hair of humans, and it has occasionally been reported as the cause of human myiasis. This condition may be more commoner than the literature suggests, because the fly is widely distributed in the northern parts of Europe and America and the infestation might pass without any ocular symptoms.'

There are two kinds of human myiasis, ophthalmic and cutaneous. Hatching occurs four to seven days after the eggs are deposited, and the larva reaches the skin by a short migration route and penetrates the tissues enzymatically. The larva may be found in the anterior (ophthalmomyiasis interna anterior) or the posterior parts (OIP) of the eye and migrate between these. Differentiation between the two conditions is justified because of considerable differences in treatment and prognosis. In OIP the larva is found in the vitreous, or intraretinally between the pigment epithelium and the neuroretina, or sometimes in the choroid. ${ }^{2-4}$ Asymptomatic cases have been recognised by the accidental finding of a dead larva in the vitreous or through hypopigmented tracks with a characteristic appearance. ${ }^{35}$ In the latter case the Correspondence to Dr M Gjötterberg, Huddinge Sjukhus, S-141 86 Huddinge, Sweden. larva had left the eye to enter the next stage in its development.

Damage to vision is caused mechanically, by a toxin, or through a uveitis which may not respond to steroid treatment. Retinal detachment may also complicate the disease. In spite of this a conservative approach has been recommended when no immediate threat to the eye is apparent. ' The following case shows that the development of the infestation is difficult to anticipate in that movements of the larva may cause damage to central parts of the fundus in a relatively short period of time.

\section{Case report}

At the beginning of September 1986 a 77 -year-old man was found to have reduced visual acuity and vitreous floaters in the left eye. He had previously been in good health and had normal vision. He had been on vacation in Lappland, one of the northern provinces of Sweden, for a few weeks during the summer. The symptoms appeared about five weeks later. The visual acuity was 0.7 in the left eye on examination. Some small vitreous opacities were seen, but otherwise there were no signs of uveitis. Criss-crossing hypopigmented tracks accompanied by haemorrhages were observed in the fundus (Fig. 


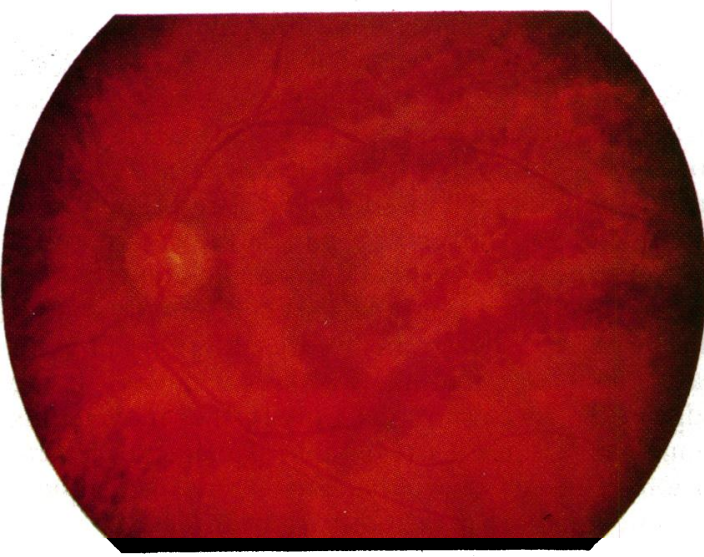

Fig. 1 The posterior pole in the left eye. Hypopigmented subretinal tracks followed by retinal haemorrhages caused by a larva of the reindeer warble fly. The patient presented with visual floaters and reduced visual acuity $(0 \cdot 7)$.

1), and slightly below the inferior temporal vascular arcade a living subretinal larva was found (Fig. 2). This was later shown to be the first stage larva of the reindeer warble fly (Oedemagena tarandi). The larva measured approximately $3 \times 1 \mathrm{~mm}$. The tracks could be followed to a hyperpigmented spot near the ora serrata in the temporal parts of the fundus where the larva probably had entered the eye (Fig. 3). A general physical examination was negative and there were no disturbances in haematology or blood chemistry.

Removal of the larva through a pars plana vitrectomy was planned to be performed three days later. However, two days after the patient's admission to hospital the larva was found at the edge of the fovea centralis (Fig. 4), causing a subretinal haemorr-

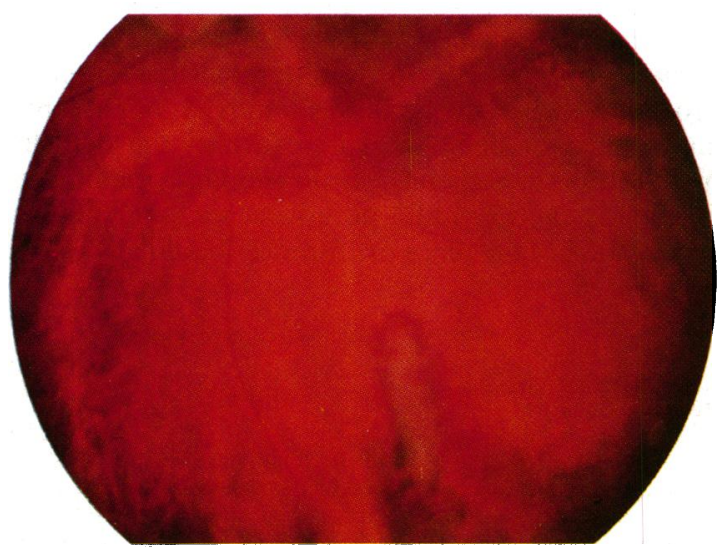

Fig. 2 Subretinal larva below the inferior temporal vascular arcade.

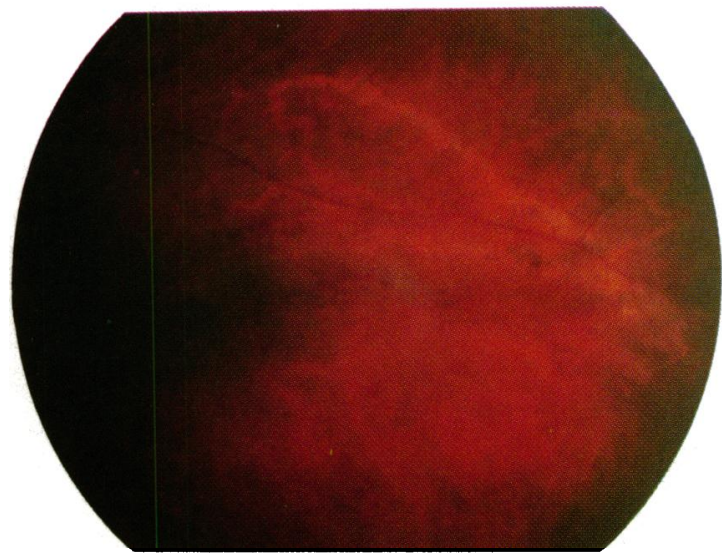

Fig. 3 Presumed place of entrance in the temporal parts of the fundus near the ora serrata.

hage with further deterioration of vision to $0 \cdot 2$. An urgent operation was performed and the larva removed alive through a retinotomy made by diathermy. Air was injected in the vitreous cavity in order to prevent retinal detachment. The retinotomy was sealed by argon laser photocoagulation the following day (Fig. 5). The haemorrhages resolved slowly in the central parts of the fundus leaving a slight retinal atrophy in the macula (Fig. 6). Vision improved to $0 \cdot 6$. A mild anterior uveitis was treated with local steroids and disappeared within a few weeks.

After about two months a small retinal detachment appeared in the temporal parts of the fundus near the ora serrata at the site where the larva had entered the eye. A small retinal break was seen. A scleral buckling procedure was carried out and the area

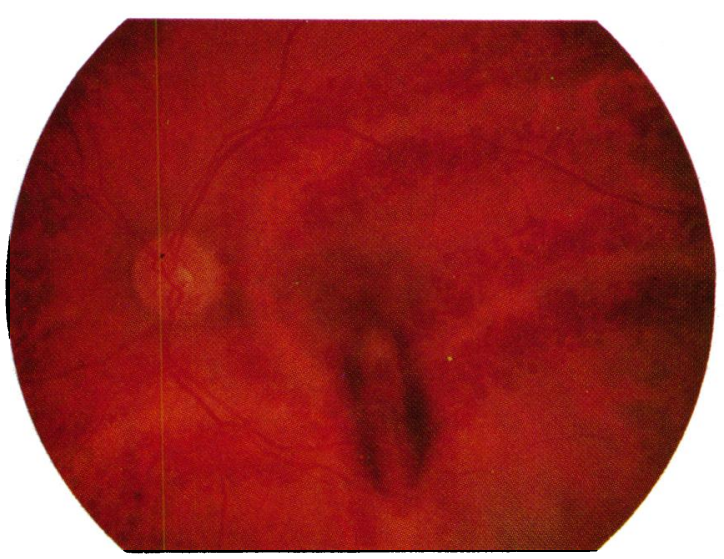

Fig. 4 Within two days the larva moved to the edge of the fovea centralis causing a subretinal haemorrhage and further reduction of the visual acuity to $0 \cdot 2$. 


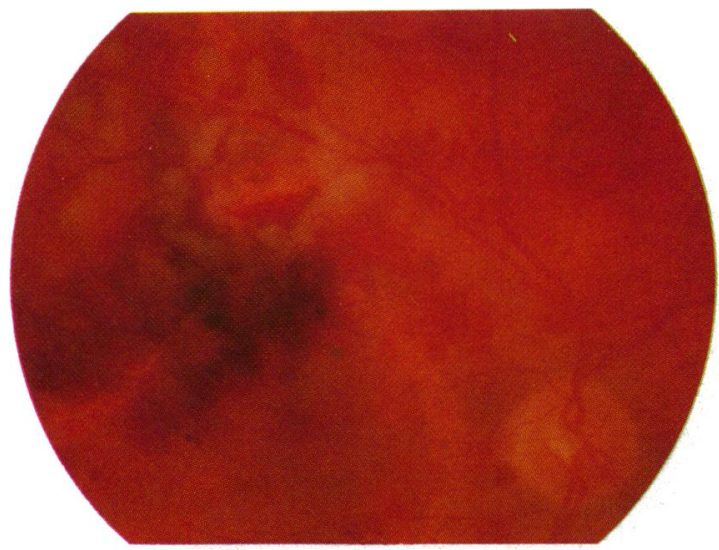

Fig. 5 Fundus after removal of the larva. The edges of the retinotomy have been sealed by argon laser photocoagulation.

treated by cryopexy. The retina became reattached and healed quickly. Central vision was not harmed by this complication.

\section{Discussion}

Syrdalen et al.' have suggested some guidelines for the treatment of OIP. According to these, the only indication for removal is when a dead larva in the vitreous causes an inflammatory reaction which does not respond to steroids. If there is no inflammation, the larva should be watched inside the eye and the development followed. A living subretinal larva should be destroyed with photocoagulation if outside the macular area, When the reindeer warble fly is the cause of OIP, it is the first larval stage that grows in

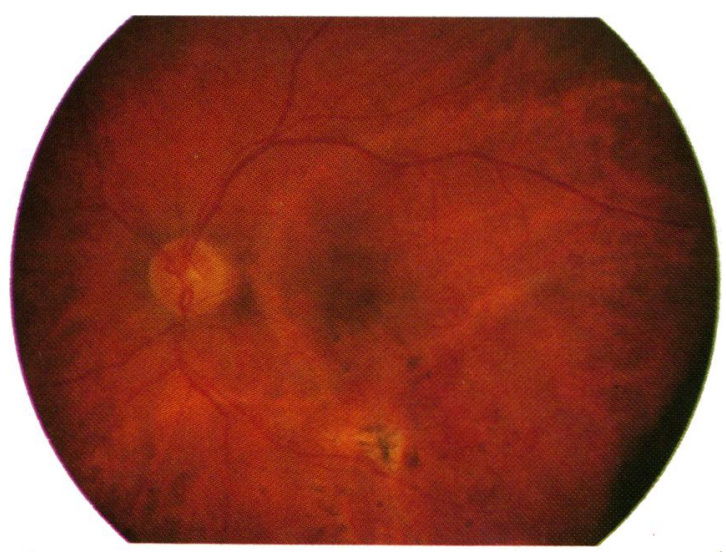

Fig. 6 Four months later. Most of the haemorrhage has resolved. Some retinal atrophy is seen in the macula, but vision has improved to $0 \cdot 6$. the eye. This stage lasts about three months, and the larva may reach a length of $9 \mathrm{~mm}$.' $^{\prime}$

When considering this and the history of our patient, we inferred that the larva had been in the eye for some weeks when it was discovered. The many tracks showed that it easily moved intraretinally, and it was probably not until it passed the central area, between the optic disc and the macula lutea, that the patient had some symptoms. When initially found, the larva was slightly below the inferior temporal vascular arcade. Within two days it moved to the edge of the fovea centralis. Any further movements would have caused irreversible damage to central vision. Thus it seems that any delay in adequate treatment entails an increased risk for the eye.

Destroying the larva with photocoagulation has been successful in some cases, ${ }^{6}$ but there is always the risk of an ensuing uveitis or damage from the release of toxic substances. In the present case photocoagulation could not be performed when the larva was found in the macula because it would have impaired central vision. However, it might be argued that the patient should have been treated with photocoagulation when he was first seen.

Pars plana vitrectomy is a safe procedure with little risk of complications. In case of OIP the larva is relatively easy to remove from the vitreous' or, as in this case, through a retinotomy from the intraretinal space. Thus in our opinion all cases of OIP should alert the clinician to consider removal as soon as possible, because the situation may quickly change and vision be damaged.

A small rhegmatogenous retinal detachment developed after two months near the site where the larva had entered the eye. Slight hyperpigmentation without any visible vitreous traction or breaks had previously been observed in the area. In spite of this, it is obvious that retinal detachment might occur after a relatively long period of time. We assume that the larva caused retinal damage when it entered the eye. However, there is a possibility that the surgical procedure, when the larva was removed, might have contributed or been the sole cause of the detachment. But, since damage from the larva cannot be excluded, caution must be advocated, and photocoagulation of the place of entrance should always be considered.

\section{References}

1 Syrdalen $\mathrm{P}$, Nitter $\mathrm{T}$, Mehl $\mathrm{R}$. Ophthalmomyiasis interna posterior: report of a case caused by the reindeer warble fly larva and review of previous reported cases. $\mathrm{Br} J$ Ophthalmol 1982; 66: 589-93.

2 Slusher MM, Holland WD, Weaver RG, Tyler ME. Ophthalmomyiasis interna posterior. Arch Ophthalmol 1979; 97: 885-7. 
3 Gass JDM, Lewis RA. Subretinal tracks in ophthalmomyiasis. Arch Ophthalmol 1976; 94: 1500-5.

4 Mason GI. Bilateral ophthalmomyiasis interna. Am J Ophthalmol 1981:91: 65-70.

5 Ziemianski MC, Lee Ky, Sabates FN. Ophthalmomyiasis interna. Arch Ophthalmol 1980; 98: 1588-9.
6 Forman AR, Cruess AF, Benson WE. Ophthalmomyiasis treated by argon-laser photocoagulation. Retina 1984; 4: 163-5.

7 Rapoza PA, Michels RG, Semeraro RJ, Green WR. Vitrectomy for excision of intraocular larva. Retina 1986; 6: 99-104.

Accepted for publication 23 April 1987. 